\title{
Stochastic Analysis of Dead-time Systems using a Hybrid Spectral Method
}

Pham Luu Trung Duong and Moonyong Lee*

\begin{abstract}
Control systems often operate in the presence of dead-time. However, in most works, these dead-time systems are studied in a deterministic manner, which have low precision and reliability. Many natural systems often suffer stochastic noise that causes fluctuations in their behavior, making their responses deviate from nominal models. Therefore, it is important to investigate such statistical characteristic of states (mean, variance, etc.) for those stochastic systems. This problem is often called statistical analysis of a system. A hybrid spectral method represents a powerful numerical tool for statistical analysis of stochastic linear system. Thus, a hybrid spectral technique is proposed for statistical analysis of the time delay system under affections of random parameters and inputs. Numerical examples are considered to demonstrate the validity of the proposed method. Comparison with the traditional Monte-Carlo and the polynomial chaos methods is made to demonstrate the computationally lessdemanding feature of the proposed method.
\end{abstract}

Keywords: Dead-time process, Monte-Carlo, operational matrix, polynomial chaos, uncertainty quantification.

\section{INTRODUCTION}

In many engineering applications, there is a need to simulate the mathematical model of process under study in the form of time delay system, especially in the process control area [1-4]. To take into account the effect of the model mismatch and unknown inputs, unknown quantities are modeled stochastically with known distributions (e.g., random variable, random process input).

The most well-known method for studying the effect of stochastic uncertainties is the sampling based methods: Monte-Carlo (MC) and Quasi Monte-Carlo (QMC) methods [5-8], which relies on the sampling of stochastic quantities according to their distributions. Since for the MC method the estimation of the mean converges with the inverse square root of the number of samples, the problem of simulating these stochastic models with low computational effort is still a challenge.

Recently, the generalized polynomial chaos (gPC) method [9-13] has been widely accepted as an alternative to $\mathrm{MC}$ thanks to its accuracy and low computational demand for systems having inputs with relatively high correlation lengths. For simulating stochastic systems with the gPC method, the random inputs of many systems involve random processes approximated by

Manuscript received October 17, 2013; revised September 22, 2014 and October 21, 2014; accepted November 2, 2014. Recommended by Associate Editor Choon Ki Ahn under the direction of Editor Hyungbo Shim.

This study was supported by a grant from the Gas Plant R\&D Center funded by the Ministry of Land, Transportation and Maritime Affairs (MLTM) of the Korean government.

Pham Luu Trung Duong and Moonyong Lee are with School of Chemical Engineering, Yeungnam University, Daedong 214-1, Gyeongsan 712-749, Korea (e-mails: pluutrungduong@yahoo. com, mynlee@yu.ac.kr).

* Corresponding author. truncated Karhunen-Loeve (KL) expansions, and the input's dimensionality depends on the correlation lengths of these processes. For input with low correlation length, the number of dimensions required for accurate representation can be large, which increases the computational demanding by the gPC method substantially.

In the recent work [12], the authors introduced a spectral method for simulating single input single output (SISO) dead-time systems with stochastic parameter uncer-tainties without considering stochastic additive input. In this work, a hybrid spectral method based on [14] is proposed for the analysis of dead-time system with a small dead-time under both stochastic parameter uncertainties and additive input. This paper is organized as follows: In Section 2, information about the operational matrix method (OP) is introduced. In Section 3 , the hybrid spectral method, which combines the advantages of stochastic collocation (SC) [10] and the operational matrix method for a time delay system is described. Numerical examples are considered in Section 4 to illustrate the usefulness of the proposed method.

\section{OPERATIONAL MATRIX: THEORY}

2.1. Orthogonal function and function approximation

Definition [15]: Orthogonal functions with respect to a weighting function $v(\mathrm{t})$.

A set of functions:

$$
\left\{\psi_{i}(t)\right\}, \quad i=1, \ldots, N
$$

are said to be orthonormal with respect to a non-negative weighting function $v(t)$ over the interval $[0, T]$ if:

$$
\int_{0}^{T} \psi_{i}(t) \psi_{j}(t) v(t) d t= \begin{cases}1, & i=j \\ 0, & i \neq j .\end{cases}
$$


Any function $\mathrm{f}(\mathrm{t})$ that can be absolutely integrated on the time interval can be expanded into a series form:

$$
f(t)=\sum_{i=1}^{\infty} c_{f_{i}} \psi_{i}(t)
$$

where $\left\{\psi_{i}(t)\right\}_{i=1}^{\infty}$ is a set of orthogonal basis function on this interval with their corresponding weight $v(\mathrm{t})$ and coefficients given by:

$$
c_{f_{i}}=\int_{0}^{T} f(t) \psi_{i}(t) v(t) d t .
$$

In practice, only the first $N$ terms of the series (4) are considered, i.e.,:

$$
f(t)=\sum_{i=1}^{N} c_{f_{i}} \psi_{i}(t)=\boldsymbol{\psi}_{N}(\mathrm{t})^{T} C_{f}
$$

with the truncated basis set and coefficient vector as:

$$
C_{f}=\left[c_{1}, \ldots, c_{N}\right]^{T} ; \boldsymbol{\psi}_{N}(t)^{T}=\left[\psi_{1}(t), \ldots, \psi_{N}(t)\right] .
$$

More details on different types of orthogonal functions can be found in $[16,17]$ and references therein.

\subsection{Orthogonal function and function approximation}

The operational matrix of integration is a square matrix obtained by integrating each element of the basis vector and expressing the result in terms of the original set of basis functions [15]:

$$
\int_{0}^{t} \boldsymbol{\psi}_{N}(\tau) d \tau=A_{i}^{T} \boldsymbol{\Psi}_{N}(t), \quad t \in[0, T]
$$

Consider the one-fold integration defined as:

$$
x(t)=\int_{0}^{t} y(\tau) d \tau
$$

Equation (8) can be expressed in terms of operational matrix of integration as in [9]:

$$
x(t)=\left(C_{x}\right)^{T} \boldsymbol{\psi}_{N}(t)=\left(A_{i} C_{y}\right)^{T} \boldsymbol{\psi}_{N}(t),
$$

where $C_{x}$ and $C_{y}$ are column vectors of coefficient expansions for $x(t)$ and $y(t)$, respectively.

Operational matrix of derivative similarly derives as:

$$
d \psi_{N}(t) / d t=A_{d}^{T} \psi_{N}(t) .
$$

Consider the following derivative in (11):

$$
y(t)=d x / d t \text {. }
$$

The derivative in (11) can be rewritten by applying derivative operational matrix as:

$$
y(t)=\left(C_{y}\right)^{T} \boldsymbol{\psi}_{N}(t)=\left(A_{d} C_{x}\right)^{T} \boldsymbol{\psi}_{N}(t) .
$$

The operational matrix of derivative and integration for each set of orthogonal functions can be obtained directly from its definitions in (7) and (10). The exact analytical formulations for these matrices for different orthogonal sets are tabulated in [16-18].

A SISO linear system can be described by the differential equation:

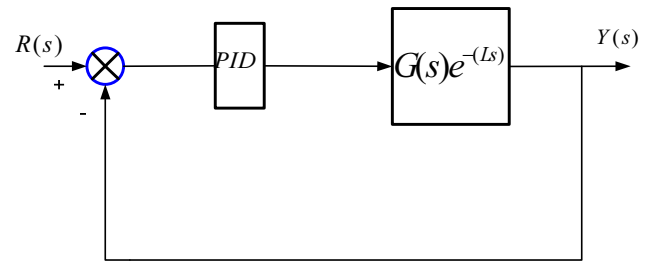

Fig. 1. Closed-loop system for a SISO dead-time system.

$$
a_{n} d^{n} u / d t^{n}+\ldots+a_{0}=b_{m} d^{m} y / d t^{m}+\ldots+b_{0}
$$

or by the transfer function:

$$
G(s)=Y(\mathrm{~s}) / \mathrm{U}(\mathrm{s})=\left(a_{n} s^{n}+\ldots+a_{o}\right) /\left(b_{m} s^{m}+\ldots+b_{0}\right) .
$$

Utilizing the operational matrix of derivatives, the system in (13) can be rewritten in terms of the operational matrix $A_{G}$ :

$$
A_{G}=\left[a_{n}\left(A_{d}\right)^{n}+\ldots+a_{0} I\right]^{-1}\left[b_{m}\left(A_{d}\right)^{m}+\ldots+b_{o} I\right],
$$

where $I$ is the identity matrix.

Thus, the spectral characteristic (or expansion coefficients) for plant's input and output are linked by:

$$
C_{y}=A_{G} C_{u} \text {. }
$$

A closed-loop control system normally involves several elements, such as a controller and plant in Fig. 1. Hence, the closed-loop's operation matrix can be found using block diagram algebra similar to the block algebra used for transfer function as described below [9]:

Consider a dead-time plant in Fig. 1:

$$
G(\mathrm{~s}) \exp (-L s) \text {. }
$$

The delay-free part of system $G(s)$ is represented by an operational matrix $A_{G}$.

Applying Pade approximation and operation matrix technique, the time delay can be represented by a operational matrix $A_{P}$.

The operational matrix for the ideal PID controller $C(s)=K_{p}+K_{i} / s+K_{d} s$ becomes $A_{P I D}=K_{p} I+K_{i} A_{i}+K_{d} A_{d}$. is:

The operational matrix for the closed-loop system thus

$$
A_{c}=\left(I+A_{L}\right)^{-1} A_{L},
$$

where $A_{L}=A_{P I D} A_{G} A_{P}$ is the loop operational matrix.

Thus, the closed-loop system has its input and output linked by:

$$
\begin{aligned}
& C_{y}=A_{c} C_{R} ; \quad R(t)=\psi(t)^{T} C_{R} ; \\
& Y(t)=\psi(t)^{T} C_{Y}=\psi(t)^{T} A_{c} C_{R} .
\end{aligned}
$$

\section{STOCHASTIC ANALYIS FOR A DEAD-TIME SYSTEM USING A HYBRID SPECTRAL METHOD}

3.1. Orthogonal function and function approximation In general, the parameters of the plant given by (14) $a_{i}$ 
and $b_{j}$ are assumed to be independent random parameters with known distribution. The input $R(t)$ is assumed to be a random process with given mean and covariance functions as $m_{R}(t)$ and $\kappa_{R}\left(t_{1}, t_{2}\right)$.

Expanding the mean in the form of (5):

$$
m_{R}(t)=E[R(t)]=\boldsymbol{\psi}_{N}(t)^{T} C_{m_{R}} .
$$

Similarly, the covariance function of the input can be expanded into a two dimensional series as:

$$
\begin{aligned}
\kappa_{R R}\left(t_{1}, t_{2}\right) & =\sum_{i=1}^{N} \sum_{j=1}^{N} \psi_{i}\left(t_{1}\right) \psi_{j}\left(t_{2}\right) c_{i j} \\
& =\boldsymbol{\psi}_{N}\left(t_{1}\right)^{T} C_{K_{R R}} \boldsymbol{\psi}_{N}\left(t_{2}\right),
\end{aligned}
$$

where the coefficient matrix $C_{\kappa_{R R}}$ is defined as:

$$
\begin{gathered}
C_{\kappa_{R R}}=\left[c_{i j}\right]_{i, j=1}^{N}=\left[\begin{array}{llll}
c_{11} \cdots & c_{1 j} \cdots & c_{1 N} \\
\vdots & \vdots & \vdots & \\
c_{N 1} \ldots & c_{N j} \cdots & c_{N N}
\end{array}\right] ; \\
c_{i j}=\int_{0}^{T} \int_{0}^{T} \kappa_{R R}\left(t_{1}, t_{2}\right) \psi_{i}\left(t_{1}\right) \psi_{j}\left(t_{2}\right) d t_{1} d t_{2} .
\end{gathered}
$$

Due to an assumption on the stochastic nature of parameters and the input, the output is a random process with spectral characteristics (coefficient expansions) of mean and covariance functions given by [9]:

$$
\begin{aligned}
& C_{m_{y}}=E\left[A_{C}\right] C_{m_{R}} ; \\
& C_{\kappa_{Y Y}}=E\left[A_{C}\left\{C_{K_{R R}}+C_{m_{R}}\left(C_{m_{R}}\right)^{T}\right\} A_{C}{ }^{T}\right],
\end{aligned}
$$

or

$$
\begin{aligned}
& m_{Y}(t)=\boldsymbol{\psi}_{N}(t)^{T} C_{m_{Y}} \\
& \kappa_{Y Y}\left(t_{1}, t_{2}\right)=\boldsymbol{\psi}_{N}\left(t_{1}\right)^{T} C_{K_{Y Y}} \boldsymbol{\psi}_{N}\left(t_{2}\right) .
\end{aligned}
$$

Random parameters of the system result in a random closed-loop operational matrix $A_{c}$ in (23) and (24), the moment of which can be estimated by a stochastic collocation method, described in the next subsection.

\subsection{Stochastic collocation for an operational matrix}

Assume that a random operational matrix has the form:

$$
A=A(\xi),
$$

where $\xi=\left(\xi_{1}, \xi_{2}, \ldots, \xi_{n}\right)$ is a vector of independent random parameters with probability density function (pdf) $\rho_{i}\left(\xi_{i}\right): \Gamma_{i} \rightarrow \mathbb{R}^{+}$. The joint pdf of $\xi$ is $\boldsymbol{\rho}=\prod_{i=1}^{n} \rho_{i}$ with the support $\Gamma \equiv \prod_{i=1}^{n} \Gamma_{i} \in \mathbb{R}^{n}$. For each random parameter $\xi_{i}$, choose a suitable quadrature set $\left\{\xi_{i}^{(m)}, w^{(m)}\right\}_{m=1}^{q_{i}}$ according to the probability density so that one-dimensional integration can be accurately approximated by:

$$
\int_{\Gamma_{i}} A\left(\xi_{i}\right) \rho_{i}\left(\xi_{i}\right) d \xi_{i}=\sum_{i=1}^{q_{i}} A\left(\xi_{i}^{(m)}\right) w_{i}^{(m)}
$$

where $\xi_{i}^{(m)}$ is the $\mathrm{m}^{\text {th }}$ node and $w^{(m)}$ is the corresponding quadrature weight for the $\mathrm{i}^{\text {th }}$ random component. Construct a multi-dimensional cubature set by tensorizing the one-dimensional quadrature set over all the combined multi-indexes $\left(j_{1}, \ldots, j_{n}\right)$. Since manipulation of the multi-indexes $\left(j_{1}, \ldots, j_{n}\right)$ is cumbersome in practice, a single index is preferable for the manipulation of these equations. The multi-index is often replaced by a graded lexicographic order index $\mathrm{j}$ [10]. Since the probability density functions are the same as the weighting functions of the cubature, the moment of the random matrix is approximated by:

$$
\begin{aligned}
E[A] & =\int_{\Gamma} A(\boldsymbol{\xi}) \boldsymbol{\rho}(\boldsymbol{\xi}) d \boldsymbol{\xi} \\
& =\sum_{j_{1}=1}^{q_{1}} \ldots \sum_{j_{n}=1}^{q_{n}} A\left(\xi_{1}^{\left(j_{1}\right)}, \ldots, \xi_{n}{ }^{\left(j_{n}\right)}\right)\left(w_{1}{ }^{\left(j_{1}\right)} \ldots w_{n}{ }^{\left(j_{n}\right)}\right) .
\end{aligned}
$$

The MATLAB suite OPQ can be used to obtain onedimensional quadrature sets and their corresponding orthogonal polynomials (polynomial chaos) with respect to different weights [19]. The sparse grid cubature can be used in (27) for system with large number of parameter uncertainty [15].

Note that the hybrid spectral method leads to a semianalytical relationship between the first two stochastic moments of the system's input and output and thus can significantly reduce computational times in comparison with other methods. Discussion about advantages and disadvantages of other methods can be referenced from [10,14].

Remark 1: $w^{(j)}$ is the cubature weight and should not be confused with the weight function of an orthogonal set $v(t)$.

Remark 2: The work in [12] considers only stochastic parameter uncertainties with standard distribution only (Gaussian and Uniform distribution), while the described method above can handle both stochastic parameter uncertainties of non-standard distribution and additive input.

Remark 3: In the standard OP method [9,20], the moments of the random matrices in (23) and (24) are approximated by Neumann series instead of using cubature (collocation). However, since a Neumann series is used to approximate a random matrix, this approach is inherently restricted to small magnitudes of uncertainties in the delay free part of system.

\subsection{Parameterization of random process for $\mathrm{MC} / \mathrm{QMC}$} and gPC methods

Statistical analysis using either MC/QMC or $\mathrm{gPC}$ methods requires the finite dimensional representation of the random process (parameterization of random process). In other words, a random process with given mean $\mu_{X}(\mathrm{t})$ and covariance $\kappa\left(t_{1}, t_{2}\right)$ :

$$
X(t)=\mu_{X}(t)+\sum_{i=1}^{d} \sqrt{\lambda_{i}} \varphi_{i}(t) \xi_{i}
$$

What truncated order $d$ should be used in (28) depends 
on the decay property of the eigenvalues in the expansion, which depends on correlation length property of the covariance function of the process. For a random process of low correlation length, hundreds of terms of the expansion (28) are required for simulating white noise, leading to a system with the dimensions of random space with an order in the hundreds.

For the gPC method, the number of the cubature nodes increases exponentially with the dimension of random space. Hence, as the dimension of random space is increased, the computational burden is increased. For the white noise input, the gPC method becomes infeasible since hundred random variables are needed.

For the MC/QMC methods, the convergence rate does not severely depend on the dimension of random space. Hence, hundreds of random variables still can be used. However, the accuracy of QMC/MC method is low.

Remark 4: For the hybrid method (proposed), this parameterization is not necessary due to algebraic relationship between input and output in (23) and (24). Hence, the proposed leads to a lower dimension in random space.

\section{EXAMPLES}

In this section, several examples are studied to show the effectiveness of the proposed method.

\section{Example 1.a: IPDT with non white noise forcing}

Consider a stochastic analysis problem for an IPDT process, $K \exp (-L s) / s$, in closed-loop feedback with a simple proportional controller: $K_{p}=1 ; K_{i}=0 ; K_{d}=0$. The gain $K$ of the system is a random variable with a triangular distribution $\operatorname{Tr}(0.5,1,1.5)$. The time delay is deterministic $L=1$. The triangular distribution is given by the distribution function [21]:

$$
\operatorname{Tr}(a, c, b)= \begin{cases}(\mathrm{x}-\mathrm{a})^{2} /((b-a)(c-a)) & \text { if } a \leq x \leq c \\ 1-(\mathrm{b}-\mathrm{x})^{2} /((b-a)(c-a)) & \text { if } c \leq x \leq b .\end{cases}
$$

The input $R(t)$ is a random process with mean $m_{R}(t)=1(t)$ and covariance $\kappa_{R R}\left(t_{1}, t_{2}\right)=0.25 \exp \left(-2 \mid t_{1}\right.$ $\left.-t_{2} \mid\right)$. For the operational matrix, a block pulse function is used for this particular example for the sake of ease of construction. A (3/3) Pade approximation was used in this example. Higher order expansion will not lead to any improvement [20]. The statistics (mean and variance) of the output estimated using the hybrid spectral method are shown in Fig. 2. The means and variances estimated by the gPC and QMC methods are also shown in this figure. Note that for the gPC and QMC methods, there is no need for Pade approximation for handling time delay. Computational times and simulation parameters for the obtained statistics of the output by these methods are all listed in Table 1. From Table 1 and Fig. 2, it can be seen that the results of the gPC method resemble those of the hybrid spectral method as the number of cubature nodes increase. However, as the number of cubature nodes increase, the computational demand also increases. The
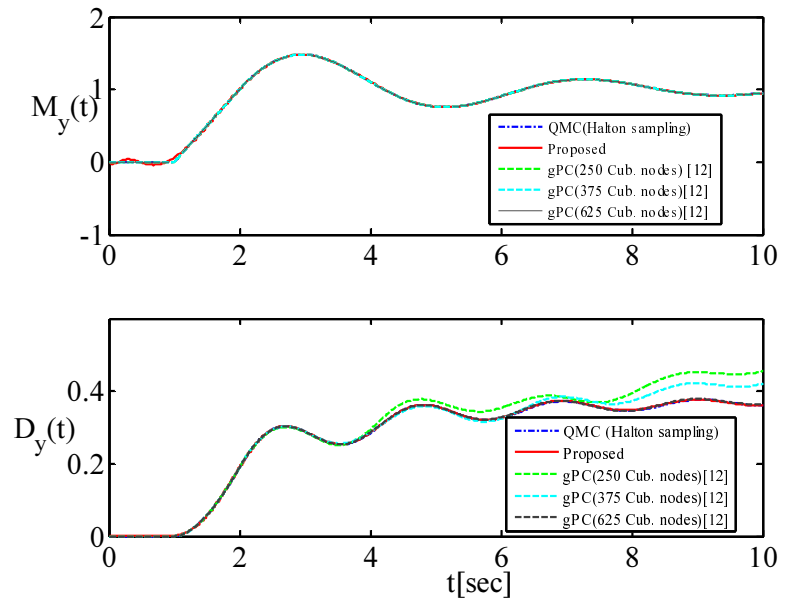

Fig. 2. Means and variances of output for Example 1.a.

MC method required the most computational effort as expected. It can be seen in the tables and figures that the hybrid spectral method gives almost the same accuracy with much less computational effort than the other methods. Thus, the hybrid spectral method can analyze IPDT systems in a computationally effective manner. For statistical analysis, the $\mathrm{gPC}$ and $\mathrm{QMC}$ require a finite dimensional representation of a random process. Noncanonical decomposition [22] is used in this example for parameterization of random process input. The number of basic functions for the proposed method can be increased until consistent result is obtained.

\section{Example 1.b: IPDT with white noise forcing}

In this example, the effect of order of Pade approximation in the proposed method is studied. Let us consider again the same IPDT system as in previous example with the same controller. Both gain and deadtime of system are now deterministic. This example was also used in [20] for showing the effect of Pade approximation for a time delay system with stochastic input.

Reference input is an ideal white Gaussian noise with zero mean and covariance $\kappa_{R R}\left(t_{1}, t_{2}\right)=\delta\left(t_{1}-t_{2}\right)$. Assume that the gain $K=1$, the exact (analytical) steady state variance of the system output is given by [23]:

$$
D_{y_{s s}}=0.5 \cos (L) /(1-\sin (L)) \text {. }
$$

Fig. 3 compares the analytical variances of system outputs under random white noise forcing and those by the proposed method with different order of Pade approximation versus the time delay $L$. The plots show that the low order Pade approximations provide a satisfactory approximation unless the time delay is somewhat significant. Note that this system does not have random parameter, hence for the proposed method the dimension of random space is zero. 1024 BPFs was used for obtaining the results by the propose method and there is no need to use the cubature since the dimension of random space is zero. It can be seen that high order Pade approximations will not lead to significant 


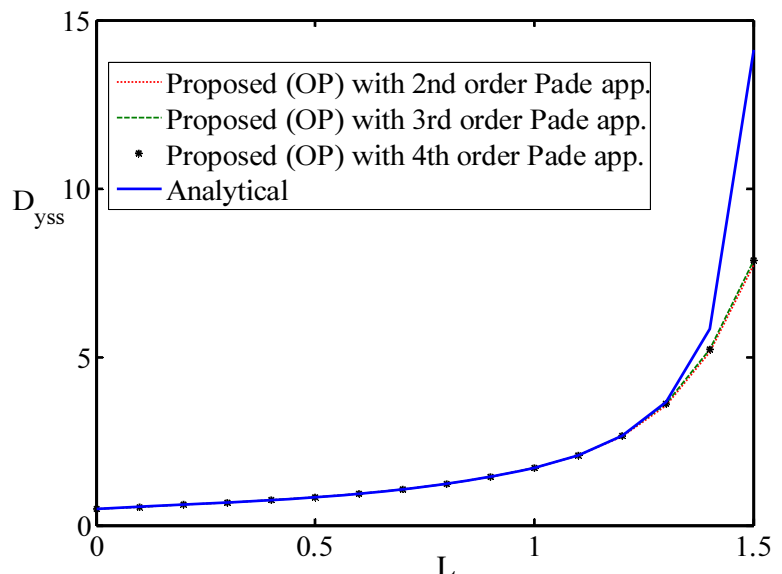

Fig. 3. Steady state variances of output as a function of time delay for Example 1.b [20].

improvement in accuracy. Hence, the (3/3) Pade approximation was used for all case studies in this work.

Since the analytical result is only available for steady state, the accuracy of the proposed method in transient time is validated by the QMC method. The gPC method becomes computational intractable for this case as explained below.

For the gPC and QMC methods, the parameterization of random input is necessary as explained in subsection 3.3. For simulation in the transient regime, ideal white noise is parameterized as in (28) with 100 terms $(d=100)$.

Thus, the dimension of random space is 100 . If 5 quadrature nodes is used per one random dimension, the gPC requires $5^{100}$ cubature nodes. This poses a numerical challenge because this means one has to simulate the system $5^{100}$ times. On the other hand, the QMC method does not severely depend on the dimension of random space. Fig. 4 shows the variances of system output for $L=0.5$. As seen from this figure, the proposed method gave quite acceptable result in both steady state and transient regimes. Note that when the number of samples is not enough (8000 samples), the QMC method yielded oscillatory result.

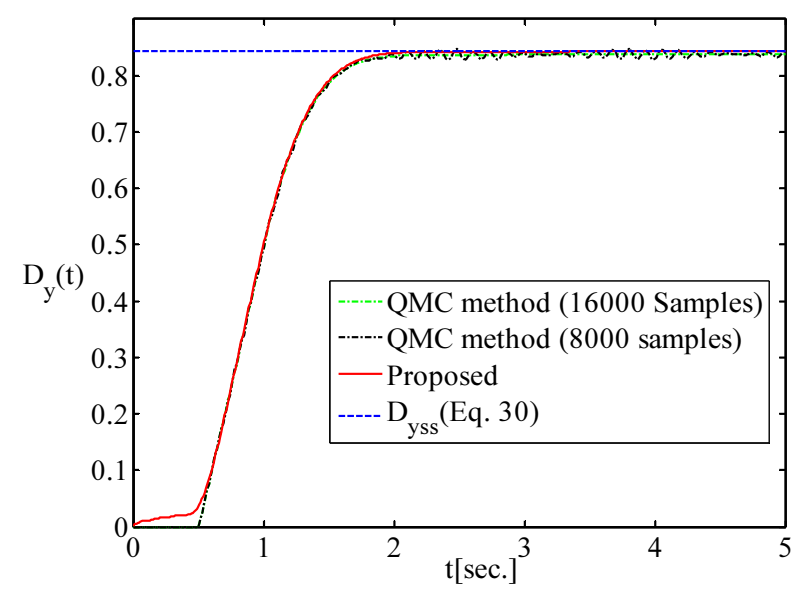

Fig. 4. Variances of system output for Example 1.b.
Remark 5: Although the convergence rate of the MC/QMC methods does not severely depend on the dimension of random space, it still depends on the magnitude of the variance of output. Hence, for the IPDT system with large time delay, when the variance rises quickly (see Fig. 3), accurate results are hardly obtained.

\section{Example 2: FOPDT system}

To demonstrate the computational efficiency of the proposed method for statistical analysis, the following FOPDT system of a heated tank in [3] was considered. An FOPDT process, $\exp (-\mathrm{Ls}) /(T \mathrm{~s}+1)$, is in closedloop feedback with a simple PI controller with $K_{p}=$ 2.5; $K_{i}=1.67 ; K_{d}=0$. The time constant $T$ and delay $L$ are random variables with triangular distributions $\operatorname{Tr}(0.5,1,1.5)$ and $\operatorname{Tr}(0,0.25,0.5)$, respectively. The system input is band-limited white noise with mean $m_{R}(t)=1(t)$ and covariance function $\kappa_{R R}\left(t_{1}, t_{2}\right)=$ $0.25 \operatorname{sinc}\left(\left(t_{1}-t_{2}\right) /(2 \pi)\right)$, where the sinc function is defined as:

$$
\operatorname{sinc}(x)=\left\{\begin{array}{l}
\sin (\pi x) /(\pi x) \text { elsewhere } \\
1 \text { for } x=0
\end{array}\right.
$$

Example 1.a showed that both gPC and proposed methods require significantly less computation to achieve a given accuracy in comparison with the traditional QMC method when the input is not white noise. Therefore this example compares these two methods only. The means and variances obtained by both methods are shown in Fig. 5. The results showed that the proposed method is capable of predicting the statistical characteristics in an accurate and effective manner.

\section{Example 3: Open loop unstable FOPDT system}

An unstable FOPDT process, $\exp (-\mathrm{Ls}) /(T \mathrm{~s}+1)$, is in the closed-loop feedback system with a simple PI controller for which: $K_{p}=-18.18 ; K_{i}=-8.18 ; K_{d}=0$. The time constant $T$ and delay $L$ are random variables with uniform distribution in the intervals $[-15,-10]$ and $[0.2,0.5]$, respectively. The input $R(t)$ is a random process with mean $m_{R}(t)=1(t)$ and covariance function
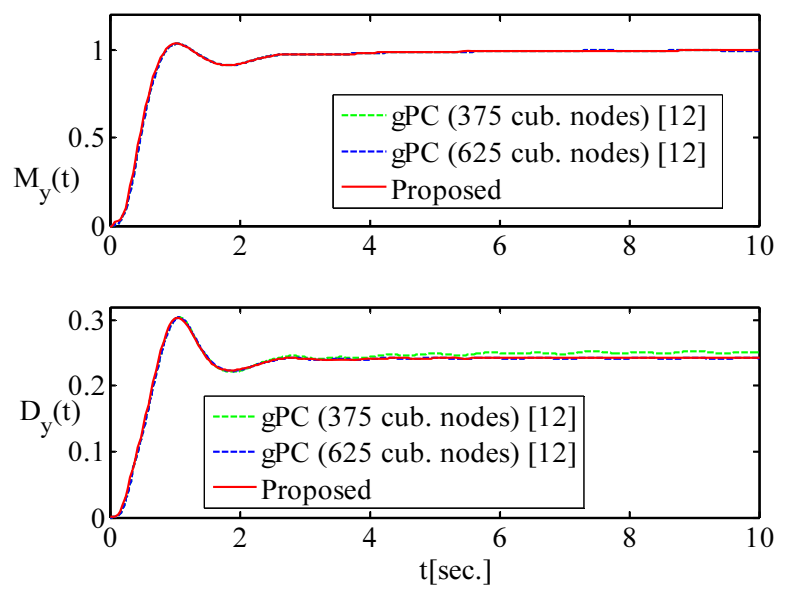

Fig. 5. Means and variances of output for Example 2. 

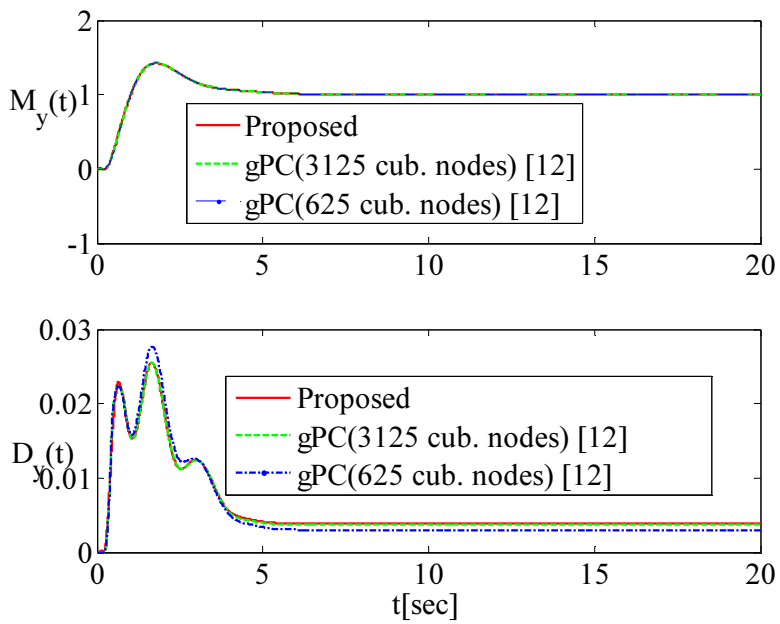

Fig. 6. Means and variances of output for Example 3.

Table 1. Computation time and simulation parameters.

\begin{tabular}{|c|c|c|c|}
\hline \multirow[b]{2}{*}{ Example } & \multicolumn{3}{|c|}{ Simulation parameters } \\
\hline & $\begin{array}{c}\text { QMC } \\
\text { (Samples) }\end{array}$ & $\begin{array}{c}\text { gPC } \\
\text { (Cub. nodes) }\end{array}$ & $\begin{array}{c}\text { Proposed } \\
\text { (No. of basis \& } \\
\text { cub. nodes) }\end{array}$ \\
\hline 1.a & 7000 & $\begin{array}{c}250,375 \\
625\end{array}$ & $512 \& 5$ \\
\hline 1.b & $\begin{array}{c}8000 \\
16000\end{array}$ & (intractable) & $1024 \& 0$ \\
\hline 2 & N/A & 375,625 & $512 \& 25$ \\
\hline 3 & N/A & 625,3125 & $1024 \& 25$ \\
\hline \multirow{2}{*}{ Example } & \multicolumn{3}{|c|}{ Computation time (sec.) } \\
\hline & QMC & gPC & Proposed \\
\hline 1.a & 409.1 & $\begin{array}{c}19.5,25.5 \\
41.0\end{array}$ & 2.8 \\
\hline 1.b & $\begin{array}{l}455.5 \\
899.9\end{array}$ & N/A & 3.1 \\
\hline 2 & N/A & $26.1,45.8$ & 14.2 \\
\hline 3 & N/A & $46.9,208.5$ & 17.5 \\
\hline
\end{tabular}

$\kappa_{R R}\left(t_{1}, t_{2}\right)=0.01 \exp \left(-10\left|t_{1}-t_{2}\right|\right)$. Note that the correlation length of input is now $1 / 10$ while in Example 1 the correlation length was $1 / 2$. The statistics of the output estimated using the proposed and gPC methods are shown in Fig. 6. Computational times and simulation parameters for the obtained statistics of the output by proposed and gPC methods are all listed in Table 1.

It is clear that if the gPC method is used with the same number of cubature nodes as in Example 2, it can not get accurate result. The same effect was observed in Examples 1.a and 2. It can be seen that the hybrid spectral method gives almost the same accuracy with much less computational effort than the gPC method.

\section{CONCLUSIONS}

A hybrid spectral method was proposed for analysis of stochastic SISO linear dead-time systems with both stochastic parameter uncertainties and additive input for the first time. The proposed hybrid method combined the advantages of operational matrix and collocation methods. It bypasses the computationally demanding parameterization of random forcing when predicting the statistical characteristics of the system output, and hence reduces the dimension of the random space. It also allows handling of systems with large parameter uncertainties, which is impossible for standard operational techniques [9]. However, due to an inherent limitation of Pade approximation, the proposed method restricts to the system with a relatively small dead-time. Since the computational need for the proposed system is substantially reduced in comparison with the MC and gPC methods, it is more suitable for the optimization of systems under the influence of stochastic perturbations, which will be incorporated in future work.

\section{REFERENCES}

[1] B. W. Bequette, Process Control: Modeling, Design and Simulation, Prentice Hall PTR, 2002.

[2] C. Brosilow and B. Joseph, Techniques of Model Based Control, Prentice Hall PTR, 1999.

[3] J. E. Normey-Rico and E. F. Camacho, Control of Dead-time Processes, Springer-Verlag, London, 2007.

[4] D. E. Seborg, T. F. Edgar, and D. A. Mellichamp, Process Dynamics and Control, 2nd edition, Wiley, New Jersey, Hoboken 2004.

[5] J. S. Liu, Monte Carlo Strategies in Scientific Computing, Springer-Verlag, 2001.

[6] K. A. Puvkov and N. D. Egupov, Eds, Classical and Modern Theory of Control System (in Russian), BMSTU Press, Moscow, 2003.

[7] L. Dunn and J. K. Shultis, Exploring Monte Carlo methods, Elsevier, 2011.

[8] D. P. Kroese, T. Taimre, and I. B. Zdravko, Handbook of Monte Carlo Methods, Wiley, New Jersey, Hoboken, 2011.

[9] K. A. Puvkov, N. D. Egupov, A. M. Makarenkov, and A. I. Trofimov, Theory and Numerical Methods for Studying Stochastic Systems (in Russian), Fizmatlits, Moscow, 2003.

[10] D. Xiu, Numerical Method for Stochastic Computation: Spectral Approach, Princeton University Press, New Jersey, 2011.

[11] D. Xiu, "Fast numerical methods for stochastic computations: a review," Commun. Comp. Physics, vol. 5, no. 2-4, pp. 242-272, 2009.

[12] P. L. T. Duong and M. Lee, "Statistical analysis of dead-time system using a deterministic equivalent modeling method," Asia-Pacific Journal of Chem. Eng., vol. 6, pp. 369-378, 2011.

[13] D. Xiu and G. E. Karniadakis, "The Wiener-Askey polynomial chaos for stochastic differential equation," SIAM J. Sci. Comput., vol. 24, no. 2, pp. 619644, 2003.

[14] P. L. T. Duong and M. Lee, "Uncertainty propagation in stochastic fractional order processes using spectral methods: a hybrid approach," Commun. Nonlinear Sci. and Numer. Simulation, vol. 17, no. 2, pp. 4262-4273, 2012.

[15] D. Xiu, "Efficient collocational approach for parametric uncertainty analysis," Commun. Comp. 
Physics, vol. 2, no. 2, pp. 293-309,2007.

[16] B. K. Datta and M. Mohan, Orthogonal Functions in System and Control, World Scientific Publishing Co. Pte. Ltd, Singapore, 1999.

[17] S. V. Lapin and N. D. Egupov, Theory of Matrix Operator and its Application in Automatic Control (in Russian), BMSTU Press, Moscow, 1997.

[18] A. H. Bhrawy, D. Baleanu, L. M. Assas, and J. A. T. Machado, "On a generalized Laguerre operational matrix of fractional integration," Math. Problems in Engineering, vol. 2013, 7 pages, Article ID 569286, 2013.

[19] W. Gaustchi, Orthogonal Polynomials: Computation and Approximations, Oxford University Press, 2003.

[20] P. L. T. Duong and M. Lee, "Robust PI controller design for integrator plus dead-time process with stochastic uncertainties using operational matrix," Korean Journal of Chem. Eng., vol. 30, pp. 19901996, 2013.

[21] C. Forbes, M. Evans, N. Hasting, and B. Peacock, Statistical Distribution, John Wiley \& Son, Hoboken, 2011.

[22] V. I. Chernhecki, Analysis Accuracy of Nonlinear Control (in Russian), Masintroenie, 1968.

[23] S. Guillouzic, I. L'Heureux, and A. Longtin, "Small delay approximation of stochastic delay differential equations," Physical Review E, vol. 59, no 4, pp. 3970-3982,1999. 